\title{
Arrested phase separation in chiral fluids of colloidal spinners
}

\author{
Helena Massana-Cid $\odot,{ }^{1,2, *}$ Demian Levis $\odot,{ }^{1,3, *}$ Raúl Josué Hernández Hernández $\odot,{ }^{1,3}$ \\ Ignacio Pagonabarraga, ${ }^{1,3,4}$ and Pietro Tierno $\oplus^{1,3,5, \dagger}$ \\ ${ }^{1}$ Departament de Física de la Matèria Condensada, Universitat de Barcelona 08028, Spain \\ ${ }^{2}$ Dipartimento di Fisica, Sapienza Università di Roma, 00185 Rome, Italy \\ ${ }^{3}$ Universitat de Barcelona Institute of Complex Systems (UBICS), Universitat de Barcelona, 08028 Barcelona, Spain \\ ${ }^{4}$ CECAM, Centre Européen de Calcul Atomique et Moléculaire, \\ École Polytechnique Fédérale de Lausanne, 1015 Lausanne, Switzerland \\ ${ }^{5}$ Institut de Nanociència i Nanotecnologia, Universitat de Barcelona, 08028 Barcelona, Spain
}

(Received 21 July 2021; accepted 5 October 2021; published 10 November 2021)

\begin{abstract}
We investigate phase separation in a chiral fluid, made of spinning ferromagnetic colloids that interact both via hydrodynamic and dipolar forces and collectively organize into separated circulating clusters. We show that, at high spinning frequency, hydrodynamics dominate over attractive magnetic interactions and impede coarsening, forcing the particles to assemble into a collection of finite rotating clusters of controllable size. We introduce a minimal particle-based model that unveils the fundamental role of hydrodynamics and the boundary plane in the self-organization process of the colloidal spinners. Our results shed light on the control of coarsening and dynamic self-assembly in chiral active systems and the key role played by fluid mediated long-range interactions.
\end{abstract}

DOI: 10.1103/PhysRevResearch.3.L042021

Coarsening and phase separation have been extensively studied in physics, chemistry, and engineering science [1]. They are commonly observed in many condensed matter systems including liquid metals [2], semiconductors [3], but also complex fluids such as emulsions [4], colloidal suspensions [5], or protein solutions [6]. Nevertheless, when considering systems driven out of equilibrium, novel kinds of structures emerge, unattainable in equilibrium conditions [7]. Active particle systems, where autonomous interacting units continuously dissipate energy exerting mechanical forces and stresses, constitute a prominent class, displaying a variety of nonequilibrium structures [8-11]. For example, motilityinduced phase separation [12] leads to a separation between a dense and a dilute phase of active particles as a result of the competition between self-propulsion and steric or excluded volume interactions.

In contrast to self-propelling particles, spinning rotors constitute another class of active particle systems that individually do not show any directed motion, but only at a collective level due to the produced hydrodynamic flow [13-16]. When torqued by an external field, the rotors present a well defined chiral state. Thus, in a continuum approximation, a dense collection of such particles can be considered as a chiral fluid [17-20]. Such systems break parity and time reversal symmetries and display emerging phenomena including a

\footnotetext{
*These authors contributed equally to this work. †ptierno@ub.edu
}

Published by the American Physical Society under the terms of the Creative Commons Attribution 4.0 International license. Further distribution of this work must maintain attribution to the author(s) and the published article's title, journal citation, and DOI. novel viscosity term called the odd (or Hall) viscosity [21,22]. In this context, a collection of spinning ferromagnetic cubes that assemble due to an in-plane rotating magnetic field has recently been proposed as an experimental model system for a chiral fluid [23-25]. While aggregation of magnetic particles [26-30] or "holes" [31,32] under rotating fields has been previously reported, considering such systems as a continuum chiral fluid provides a complementary perspective to the field. However, such an approach neglects the effect of hydrodynamic interactions (HIs) between the individual units which effectively stir and mix fluid due to their continuous rotary motion. Thus, the kinetics and the effect of HIs on the collective organization remain still challenging puzzles that need to be addressed.

Here we investigate the collective behavior of a chiral system composed of spinning magnetic rotors that strongly interact via HIs. We show that, in contrast to the standard coarsening scenario observed for attractive particles under a rotating in-plane field [28,32,34], HIs impede coarsening for fast enough spinning and induce a phase characterized by the presence of rotating finite clusters, whose size can be controlled by the field frequency. At low spinning frequency, particles form large aggregates (following a coarsening process) which display circulating edge currents with a constant penetration depth and an edge velocity that decreases with increasing the cluster size. These observations are in line with parallel numerical simulations, which unveil the important role of the boundary plane and HIs on the collective rotor organization and the selection of a finite length scale.

Our spinning colloids are ferromagnetic hematite ellipsoids with long (short) axis equal to $b=1.8 \mu \mathrm{m} \quad(a=$ $1.3 \mu \mathrm{m})$ and a permanent magnetic moment $m=2.3 \times$ $10^{-16} \mathrm{Am}^{2}$ oriented perpendicular to $b$. More details on the particle synthesis and on the measurement of $m$ can be found 

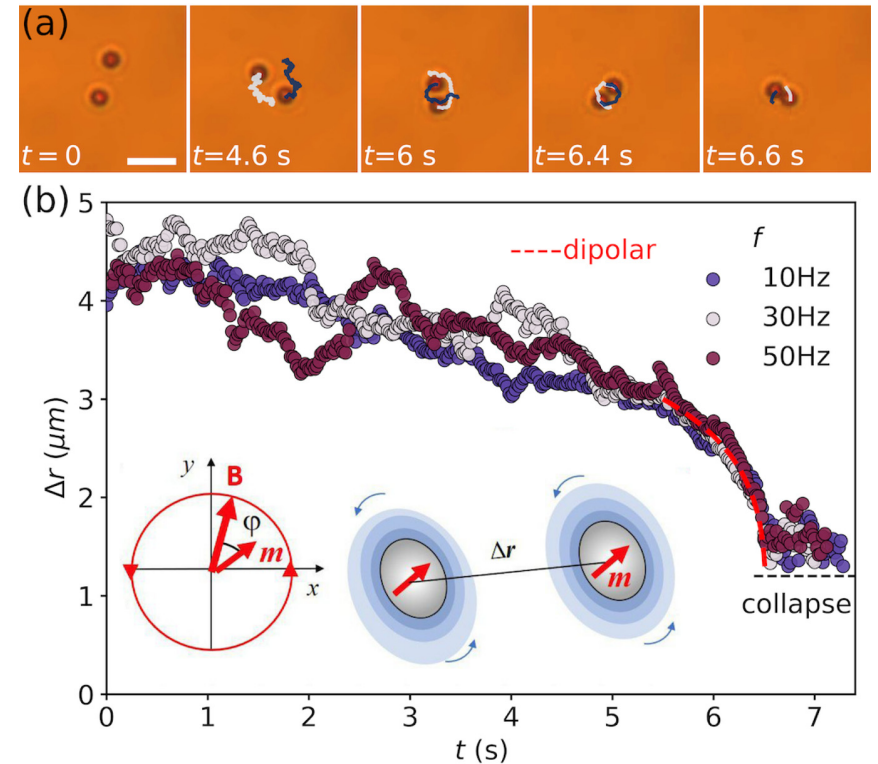

FIG. 1. (a) Sequence of images showing the collapse of a pair of spinning rotors assembled by the rotating field with frequency $f=30 \mathrm{~Hz}\left(B_{0}=3 \mathrm{mT}\right)$. The scale bar is $5 \mu \mathrm{m}$, see VideoS1 in the Supplemental Material [33]. (b) Interparticle distance $\Delta r$ between two rotors versus time $t$ for three frequencies. The dashed red line denotes a nonlinear regression considering only dipolar interactions. From particle tracking we extract the net force $\boldsymbol{F}=$ $\gamma \boldsymbol{v}_{r} / 2$ of a pair of rotors approaching at a speed $\boldsymbol{v}_{r}$, being $\gamma$ the Stokes coefficient, and obtain $\Delta r=\sqrt[1 / 5]{\Delta r_{0}^{5}-5 \alpha t}$, being $\Delta r_{0}=$ $3 \mu \mathrm{m}$ and $\alpha=3 \mu_{0} \mathrm{~m}^{2} /(16 \pi \gamma)=47.5 \pm 0.2 \mu \mathrm{m}^{5} \mathrm{~s}^{-1}$. Inset shows a schematic representation of two particles with permanent moments $\boldsymbol{m}$ under the rotating field $\boldsymbol{B}$.

in Ref. [35]. The particles are dispersed in highly deionized water and functionalized with sodium dodecyl sulfate (SDS) by adding $0.12 \mathrm{~g}$ of SDS in $80 \mathrm{ml}$ of $\mathrm{H}_{2} \mathrm{O}$. This surfactant is used to avoid irreversible aggregation due to Van der Walls interactions. We also adjust the $\mathrm{pH}$ of the resulting solution to $8.5-9.5$ by adding tetramethylammonium hydroxide. The hematite rotors sediment above a glass plate due to density mismatch, where they remain confined showing weak thermal motion. We visualize the particles using an upright optical microscope (Eclipse Ni-U, Nikon) with magnification objectives $40 \times, 60 \times$ and equipped with a CCD camera (Scout scA640$74 \mathrm{f}$, Basler) working at $50 \mathrm{fps}$. We use a set of custom made magnetic coils arranged on the microscope stage to apply time dependent magnetic fields, using a wave generator (AimTTi TGA1244) controlling a power amplifier (IMG STA-800, stage line). We limit our experiments to less than $1 \mathrm{~h}$ to avoid heating of the magnetic coil system.

We spin the hematite particles with a rotating magnetic field circularly polarized in the $(\hat{\boldsymbol{x}}, \hat{\boldsymbol{y}})$ plane, with frequency $f$ and amplitude $B_{0}, \boldsymbol{B} \equiv B_{0}(\cos (2 \pi f t) \hat{\boldsymbol{x}}+\sin (2 \pi f t) \hat{\boldsymbol{y}})$. Each particle is subjected to a magnetic torque $\tau_{m}=|\boldsymbol{m} \times \boldsymbol{B}|=$ $m B \sin \varphi, \varphi$ being the phase lag angle between $\boldsymbol{m}$ and $\boldsymbol{B}$, see inset in Fig. 1(b). The torque balance equation in the overdamped limit, $\tau_{m}+\tau_{v}=0$, determines the single rotor dynamics, where $\tau_{v}=-\zeta_{r} \dot{\beta}$ is the viscous torque, $\beta=\omega t-$ $\varphi$, and $\zeta_{r}$ the rotational friction of the ellipsoid in water. We find the critical frequency $f_{c}=m B_{0} /\left(2 \pi \zeta_{r}\right)$ that bridges the synchronous rotation from the phase slip motion. We spin always our particles at frequencies $f<f_{c}=60 \mathrm{~Hz}$ and at fixed $B_{0}=3 \mathrm{mT}$, see Fig. 1 in the Supplemental Material [33]. Thus, the rotors are always phase locked with the rotating field, $\sin \varphi=f / f_{c}$, and generate the maximum hydrodynamic flow due to their high spinning. For large $f$, we can approximate such a flow as the one generated by a rotating spherical particle, $\boldsymbol{u}=\frac{\tau_{m}}{8 \pi \eta r^{2}} \boldsymbol{z} \times \hat{\boldsymbol{r}}$, which is purely azimuthal and decays as $1 / r^{2}[36,37]$. Thus, the strength of HIs will directly depend on $f$ and can be separately tuned in our system.

Figure 1(a) shows the collapse dynamics of a pair of interacting rotors when initially placed at a distance $\sim 5 \mu \mathrm{m}$. The pair shows initially a relative orbital motion and finally collapse into a rotating dimer after $6.5 \mathrm{~s}$. The dynamics results from the interplay between HIs and magnetic attractions. In the absence of the latter, the two rotors initially placed at a distance $\Delta r_{0}$ would form a stable rotating pair and rotate around their center of mass with an angular velocity $\boldsymbol{\omega}=$ $\tau_{m} /\left(4 \pi \eta \Delta r_{0}^{3}\right) \hat{z}$. However, magnetic dipolar attractions reduce the relative distance $\Delta \boldsymbol{r}$ until becoming completely dominant at a separation of $\sim 3 \mu \mathrm{m}$, Fig. 1(b). For two point dipoles $(i, j)$ with equal moments $\boldsymbol{m}$ and at a separation $\boldsymbol{r}$, such interactions can be time averaged over several field cycles and become attractive, described by the effective potential $\left\langle U_{m}\right\rangle=$ $-\frac{\mu_{0} m^{2}}{8 \pi|r|^{3}}$, where $\langle\ldots\rangle$ denotes the time average, and $\mu_{0}=4 \pi \times$ $10^{-7} \mathrm{Hm}$ [38]. Thus, these interactions are independent on $f$, in contrast to HIs. At large frequency $(f=30 \mathrm{~Hz})$ the separation distance between the pair $\Delta r$ displays a series of oscillations that results from the interplay between the dipolar attraction and the repulsive squeezed flow generated by the rotating particles. The amplitude of these oscillations, however, decreases with $f$ since the strength of the generated flow is reduced, Fig. 1(b). We use a range of frequency where raising $f$ increases the strength of HIs $\left(\sim 1 / r^{2}\right)$, while dipolar attractions $\left(\sim 1 / r^{3}\right)$ remain unchanged.

We next investigate the collective assembly of the magnetic spinners by varying the driving frequency. As shown in Fig. 2(a), for $f=10 \mathrm{~Hz}$ we find that the normalized cluster radius, $\ell(t)=L(t) / a$, exhibits a power-law growth with exponent $1 / 3$, as expected for diffusive systems with negligible HIs $[1,39,40]$. However, the situation already changes by increasing $f$ to $f=20 \mathrm{~Hz}$, where the rotors organize into smaller clusters that do not coarsen but they grow up to a finite characteristic length $\ell_{s}$, see Fig. 2(b). Upon raising $f$, the average size of the clusters reduces, in a way compatible with the simple dependence $\ell_{s} \propto f^{-1}$. The phenomenon may be reminiscent to the dynamic clustering observed in self-propelled particle systems, where a stationary state of finite size clusters is observed [41-46]. In particular, studies of (dry) models of chiral active particles with velocity alignment, also feature phase separation at small frequencies (here describing an active torque) and a distribution of finite-size rotating clusters at larger ones, whose size is also proportional to the inverse of the frequency $[47,48]$. However, despite the intriguing similarity at this level, important differences should be highlighted between these systems and the present one, not only at the level of its microscopic constituents. Spinning colloids, once in a cluster, are not observed to leave it on short 

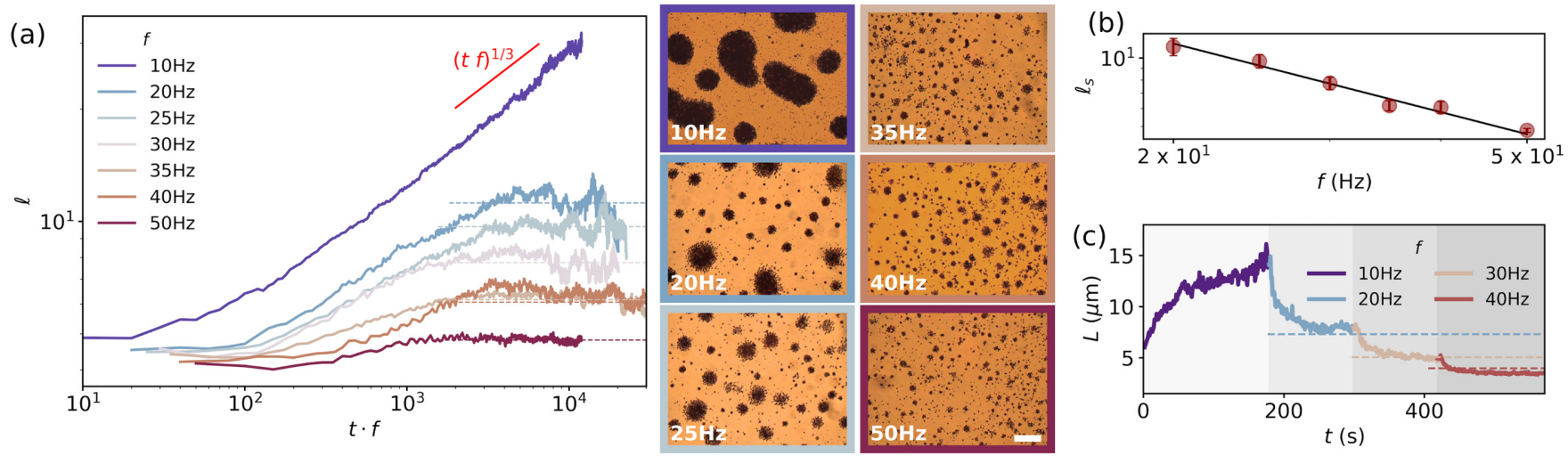

FIG. 2. (a) Time evolution of the normalized cluster radius $\ell=L / a$ for different frequencies. The left panels show the corresponding optical microscopy images of the stationary states observed at different $f$. Scale bar in the last image is $50 \mu \mathrm{m}$. See VideoS2 in Ref. [33]. (b) Stationary length $\ell_{s}$ vs $f$. Continuous line corresponds to $\ell_{s} \sim 1 / f$. (c) Quench experiment: change of the average cluster size versus time by varying the driving frequency from $f=10 \mathrm{~Hz}$ to $f=40 \mathrm{~Hz}$. Horizontal dashed lines in (a) and (c) correspond to stationary cluster sizes obtained while applying constant frequency [experiments in Fig. 2(a)].

time scales. Instead, the rotors located at the outer shell of the cluster form a circulating edge current, while the central part displays a relative slow, solid body rotation (see Fig. 3). Due to the high density of packed hematite particles in the bulk, we could not resolve whether the rotors crystallize in the bulk of large clusters. However, for smaller clusters we find that shape anisotropy of the spinning rotors frustrate ordering, which is different than the compact aggregate observed with spherical magnetic particles $[28,29,49]$. Further, to confirm that finite cluster sizes are stationary states, we perform several quenches by suddenly changing $f$ [see Fig. 2(c)]. We first set the driving frequency to $f=10 \mathrm{~Hz}$, which induces a slow coarsening towards the formation of a large cluster. We then switch the frequency to $f=20 \mathrm{~Hz}$ and observe how the system breaks into smaller rotating clusters with an average cluster size which relaxes to lower values (of area $A \sim 167 \mu \mathrm{m}^{2}$ ). A further reduction in size is observed by
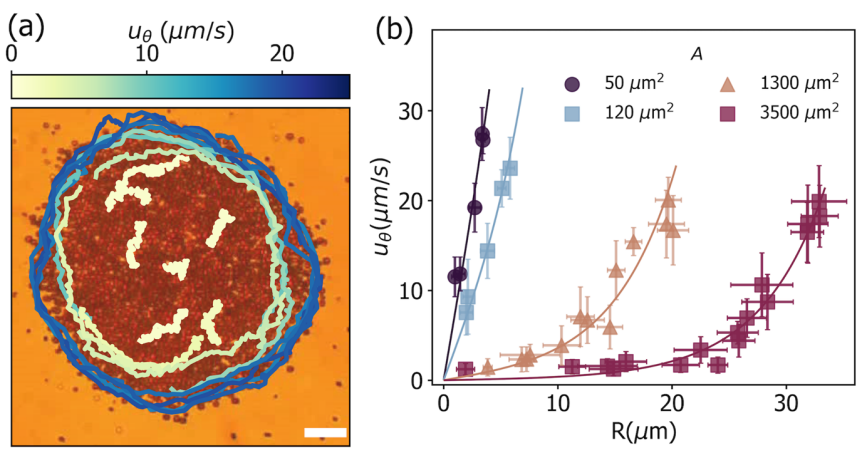

FIG. 3. (a) Microscope image of a rotating cluster in the steady state with an area $A=3500 \mu \mathrm{m}^{2}(f=10 \mathrm{~Hz})$. The trajectories of few spinners are superimposed to the image. Color bar on the top corresponds to the tangential velocity $u_{\theta}$. Scale bar is $10 \mu \mathrm{m}$. See VideoS3 in Ref. [33]. (b) Mean tangential velocity of the spinners $u_{\theta}$ versus radial distance $R$ from the center for clusters with different areas. Scattered points are experimental data; continuous lines are multiple fits using Eq. (1) by keeping constant $\alpha$ for all curves and varying the penetration depth $\delta$. quenching to $f=30 \mathrm{~Hz}\left(A \sim 80 \mu \mathrm{m}^{2}\right)$ and $40 \mathrm{~Hz}(A \sim$ $\left.49 \mu \mathrm{m}^{2}\right)$, respectively. The sizes obtained through this quenching protocol perfectly match the stationary values obtained for each frequency in Fig. 2(a), showing how $f$ controls the pattern selection. Overall, these results remark the fundamental role of HIs in the clustering process, since increasing $f$ raises the strength of the flow field generated by the spinning rotors.

In the phase separated regime, the assembled clusters display edge currents where fast spinners circulate around a slower compact particle region, as shown in Fig. 3(a). From particle tracking, we measure the tangential velocity $u_{\theta}$ for clusters having different sizes and find that it rapidly decreases towards the cluster bulk after a few layers, Fig. 3(b). For a cluster of radius $R$ composed of particles spinning at fixed angular frequency $\omega, u_{\theta}$ can be calculated by solving the hydrodynamic equations of motion considering the cluster as a continuum medium $[21,23,50]$. The resulting expression in a polar coordinate system $(r, \theta)$ centered within the cluster is given by

$$
u_{\theta}(r)=2 \omega \delta \frac{I_{1}(r / \delta)}{\alpha I_{2}(R / \delta)+I_{0}(R / \delta)},
$$

where $I_{1}$ is the modified Bessel function of the first type, $\delta$ is the penetration depth of the edge current, and $\alpha=\eta / \eta_{R}$ the ratio between the shear $(\eta)$ and the rotational $\left(\eta_{R}\right)$ viscosity of the cluster. From the data analysis in Fig. 3(b) we find the ratio $\alpha=6.2$ with a small variation of the penetration depth from $\delta=4.9 \mu \mathrm{m}$ for the smallest cluster $A=50 \mu \mathrm{m}^{2}$ to $\delta=5.3 \mu \mathrm{m}$ for the largest one $A=3500 \mu \mathrm{m}^{2}$. Such a value of $\delta$ corresponds approximately to $\sim 2$ particle layers. The corresponding edge velocity increases linearly as the cluster size decreases, varying from from $30.3 \mu \mathrm{m} \mathrm{s}^{-1}$ $(R=3.9 \mu \mathrm{m})$ to $20.5 \mu \mathrm{m} \mathrm{s}^{-1}(R=33.4 \mu \mathrm{m})$ due to the corresponding increase of the magnetic interactions with the number of particles, which favor compact structure and effectively decrease the rotational motion of the spinning particles.

To understand the mechanisms underlying spinning colloidal assembly, we adopt a particle-based approach rather than a phenomenological hydrodynamic description $[23,51]$. 
We use a minimal model that focuses on three main physical ingredients: (i) rotations at a fixed rate, (ii) isotropic attractive interactions with a short-range steric repulsion, and (iii) low Reynolds number hydrodynamics. We consider $N$ spheres located at $\boldsymbol{r}_{i}(t)=\left(x_{i}(t), y_{i}(t), h\right)$ at time $t$, moving in a $L \times L$ plane parallel to a solid wall at a distance $h$ and spinning at a constant angular velocity $\omega$. We apply periodic boundary conditions in the $z=h$ plane, where particles move accordingly to the noiseless overdamped dynamics, $\gamma \dot{\boldsymbol{r}}_{i}(t)=\boldsymbol{u}\left(\boldsymbol{r}_{i}(t)\right)+$ $\boldsymbol{F}_{i}(t)$, where $\boldsymbol{F}_{i}=-\sum_{j \neq i} \nabla_{i} V\left(r_{i j}\right)$ derives from a LennardJones potential $V(r)=4 \varepsilon\left\{(\sigma / r)^{12}-(\sigma / r)^{6}\right\}$ and $\gamma=3 \pi \eta \sigma$, where $\sigma / 2=a_{0}$ is the effective radius of the particles and $\eta$ the viscosity of the medium. HIs are taken into account by $\boldsymbol{u}\left(\boldsymbol{r}_{i}\right)$, the flow field at $\boldsymbol{r}_{i}$, due to all the other colloids and described by means of the Blake propagator, accounting for the field due to a localized force and torque in the vicinity of an infinite stationary plane with no-slip boundary conditions [52,53]. Each colloid is treated as a point from which a force and torque originates (due to pair-wise interactions between colloids and their spinning, respectively), creating a flow. The flow field generated by each particle can be decomposed into two terms, $\boldsymbol{u}=\boldsymbol{u}_{S}+\boldsymbol{u}_{R}$, arising from the force and torque singularity (Stokeslet and Rotlet, respectively). The components parallel to the wall decay as $\boldsymbol{u}^{S} \sim \frac{F}{\eta} r^{-1}$ and $\boldsymbol{u}^{R} \sim a_{0}^{3} \omega r^{-2}$, giving rise to long-range interactions in the plane, as detailed in Ref. [33].

We express time and length in units of $1 / \omega$ and $a_{0}$, respectively, leading to the dimensionless quantities: $\rho=N a_{0}^{2} / L^{2}$, the particle density, and $\Lambda=\omega a_{0}^{2} \gamma / \varepsilon$, the reduced frequency, comparing the spinning rate with the potential energy scale. Figure 4(a) displays the time evolution of the average cluster radius $\ell(t)$ at fixed $\rho=0.1, \gamma=1$, and $N=25^{2}$, starting from a random distribution of particles with zero velocity (see Ref. [33] for details). For small $\Lambda$, when attractions dominate, and fixed $h=1$, colloids aggregate into clusters that grow, eventually leading to a macroscopic condensation at long times, characterized by a $\ell(t) \sim t^{1 / 3}$ growth at long times but a slower one $\ell(t) \sim t^{1 / 6}$ at shorter times. Upon increasing $\Lambda$ (from $\Lambda \gtrsim 50$ ), chiral flows start to dominate, frustrating the tendency to form macroscopic clusters and stabilizing finitesize ones, in agreement with experiments (see Fig. 2). For the values of $\Lambda \gtrsim 50$ explored, we find finite-size clusters of similar characteristic length $\ell_{s} \approx 10 a_{0}$ (i.e., composed of around 35 particles). Late-time snapshots of the system $\left(t=6.10^{5}\right)$ where each particle is colored according to its instantaneous velocity depicted in Figs. 4(c) and 4(d) clearly highlight the change in behavior as the frequency is increased.

To highlight to what extent HIs are key in the present structure formation scenario, we now analyze more carefully the impact of a solid wall at a distance $h$ from the particles. Indeed, the flow fields generated by pointlike forces and torques are strongly affected by the presence of a wall, as it introduces a hydrodynamic screening that becomes more important as the wall is closer. For $h \gtrsim 5$, dynamic clusters develop in a regime where particles can move easily among them but do not aggregate at short times until a large cluster emerges at longer times [see Fig. 4(e)]. In the limit of an infinitely large structure, particles at its edge can move arbitrary fast. The presence of a solid wall in the vicinity of the suspension limits
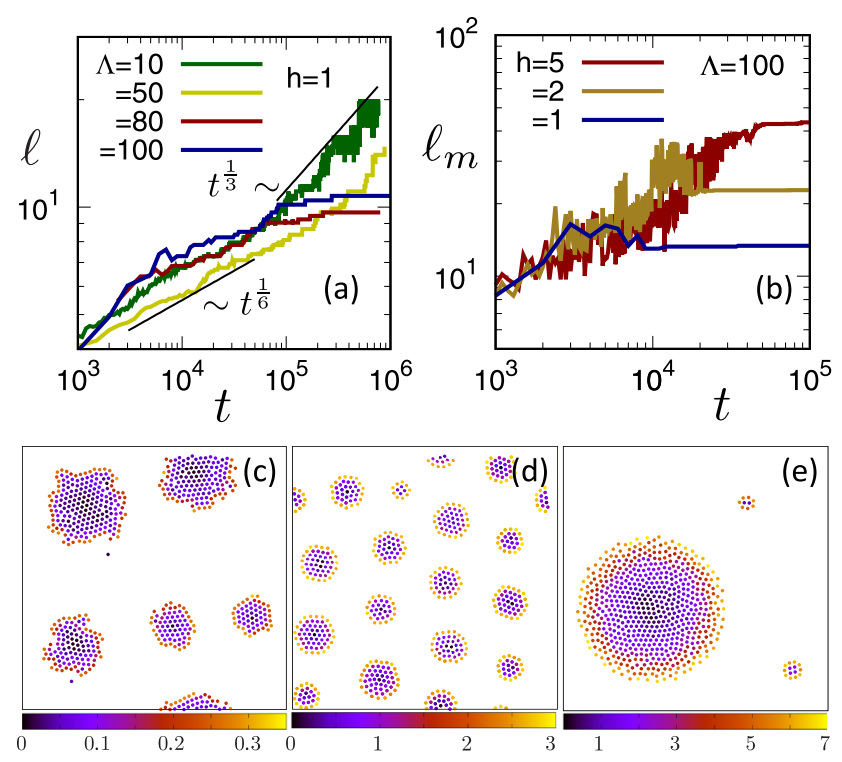

FIG. 4. Simulation results. (a) Time evolution of the average cluster radius $\ell(t)$ for $h=1$ and $\Lambda=10,50,80,100$. For slow spinning the clusters grow indefinitely, showing a slow $t^{1 / 6}$ growth at intermediate times and a long-time behavior compatible with $\ell \sim$ $t^{1 / 3}$. Faster spinning interrupts this growth, inducing the formation of clusters of finite size. (b) Time evolution of the size of the largest cluster $\ell_{m}(t)$ for fixed $\Lambda=100$ but different $h$. Corresponding latetime snapshots obtained for $h=1$ with (c) $\Lambda=10$ and (d) $\Lambda=100$, and for (e) $h=5$ with $\Lambda=100$. Each particle is colored according to its velocity, following the scale given in the bars. See VideoS4 in Ref. [33].

such fast rotation, acting as a momentum sink, and it is thus at the origin of the arrested growth of particle aggregates. The hydrodynamic friction induced by the wall, at large $\Lambda$, prevents the development of macroscopic structures, such as the one in Fig. 4(e). Instead, finite structures, whose size grows with $h$, arise. To monitor this process, we follow the evolution of the average radius of the largest cluster $\ell_{m}(t)$, see Fig. 4(b). The data show that $h$ plays a key role: Reducing $h$ also reduces the sizes of the emerging clusters. The separation from the wall thus provides, together with the driving frequency, yet another route to control colloidal spinners' self-assembly.

In summary, we have studied a chiral active fluid made of an ensemble of magnetic spinning particles and show that it phase separates to finite size clusters due to hydrodynamic interactions. Similar effects may be observed in other chiral fluids, when the rotational motion is such that the generated hydrodynamic flow plays an important role in the clustering process. Further, the possibility to control and select the cluster size with the driving frequency may provide strategies to control pattern formation in active matter systems and may be extended to other collective spinner systems, including both synthetic [54-58] and biological [59-64] ones.

D.L. acknowledges MCIU/AEI/FEDER for financial support under Grant Agreement No. RTI2018-099032J-I00. I.P. acknowledges support from MCIU (No. 
PGC2018-098373-B-100 AEI/FEDER-EU), AGAUR (No. 2017SGR-884), and Swiss National Science Foundation (No. 200021-175719). This project has received funding from the European Research Council (ERC) under the European
Unions Horizon 2020 research and innovation programme (Grant Agreement No. 811234). P.T. acknowledges support from MCIU (No. PID2019-108842GB-C21), AGAUR (2017-SGR-1061), and the Program "ICREA Acadèmia."
[1] A. Onuki, Phase Transition Dynamics (Cambridge University Press, Cambridge, 2007).

[2] C. Misbah, O. Pierre-Louis, and Y. Saito, Crystal surfaces in and out of equilibrium: A modern view, Rev. Mod. Phys. 82, 981 (2010).

[3] J. Stangl, V. Holý, and G. Bauer, Structural properties of selforganized semiconductor nanostructures, Rev. Mod. Phys. 76, 725 (2004)

[4] J. Bibette, F. Leal Calderon, and P. Poulin, Emulsions: Basic principles, Rep. Prog. Phys. 62, 969 (1999).

[5] B. Li, D. Zhou, and Y. Han, Assembly and phase transitions of colloidal crystals, Nat. Rev. Mater. 1, 15011 (2016).

[6] C. P. Brangwynne, P. Tompa, and R. V. Pappu, Polymer physics of intracellular phase transitions, Nat. Phys. 11, 899 (2015).

[7] M. C. Cross and P. C. Hohenberg, Pattern formation outside of equilibrium, Rev. Mod. Phys. 65, 851 (1993).

[8] M. C. Marchetti, J. F. Joanny, S. Ramaswamy, T. B. Liverpool, J. Prost, M. Rao, and R. A. Simha, Hydrodynamics of soft active matter, Rev. Mod. Phys. 85, 1143 (2013).

[9] C. Bechinger, R. Di Leonardo, H. Löwen, C. Reichhardt, G. Volpe, and G. Volpe, Active particles in complex and crowded environments, Rev. Mod. Phys. 88, 045006 (2016).

[10] L. P. Dadhichi, A. Maitra, and S. Ramaswamy, Origins and diagnostics of the nonequilibrium character of active systems, J. Stat. Mech. (2018) 123201.

[11] G. Gompper, R. G. Winkler, T. Speck, A. Solon, C. Nardini, F. Peruani, H. Lwen, R. Golestanian, U. B. Kaupp, L. Alvarez, T. Kiørboe, E. Lauga, W. C. K. Poon, A. DeSimone, S. MuiñosLandin, A. Fischer, N. A. Sker, F. Cichos, R. Kapral, P. Gaspard et al., The 2020 motile active matter roadmap, J. Phys.: Condens. Matter 32, 193001 (2020).

[12] M. E. Cates and J. Tailleur, Motility-induced phase separation, Annu. Rev. Condens. Matter Phys. 6, 219 (2015).

[13] I. Llopis and I. Pagonabarraga, Dynamic regimes of hydrodynamically coupled self-propelling particles, Europhys. Lett. 75, 999 (2006).

[14] M. Leoni and T. B. Liverpool, Dynamics and interactions of active rotors, Europhys. Lett. 92, 64004 (2010).

[15] N. H. P. Nguyen, D. Klotsa, M. Engel, and S. C. Glotzer, Emergent Collective Phenomena in a Mixture of Hard Shapes Through Active Rotation, Phys. Rev. Lett. 112, 075701 (2014).

[16] Z. Shen and J. S. Lintuvuori, Hydrodynamic clustering and emergent phase separation of spherical spinners, Phys. Rev. Research 2, 013358 (2020).

[17] B. C. van Zuiden, J. Paulose, W. T. M. Irvine, D. Bartolo, and V. Vitelli, Spatiotemporal order and emergent edge currents in active spinner materials., Proc. Natl. Acad. Sci. USA 113, 12919 (2016).

[18] C. Scholz, M. Engel, and T. Pöschel, Rotating robots move collectively and self-organize, Nat. Commun. 9, 931 (2018).

[19] M. Workamp, G. Ramirez, K. E. Daniels, and J. A. Dijksman, Symmetry-reversals in chiral active matter, Soft Matter 14, 5572 (2018).
[20] M. Han, M. Fruchart, C. Scheibner, S. Vaikuntanathan, W. Irvine, J. de Pablo, and V. Vitelli, Statistical mechanics of a chiral active fluid, arXiv:2002.07679.

[21] D. Banerjee, A. Souslov, A. G. Abanov, and V. Vitelli, Odd viscosity in chiral active fluids, Nat. Commun. 8, 1573 (2017).

[22] T. Markovich and T. C. Lubensky, Odd Viscosity in Active Matter: Microscopic Origin and 3d Effects, Phys. Rev. Lett. 127, 048001 (2021).

[23] V. Soni, E. S. Bililign, S. Magkiriadou, S. Sacanna, D. Bartolo, M. J. Shelley, and W. T. M. Irvine, The odd free surface flows of a colloidal chiral fluid, Nat. Phys. 15, 1188 (2019).

[24] O. Petrichenko, G. Kitenbergs, M. Brics, E. Dubois, R. Perzynski, and A. Cēbers, Swarming of micron-sized hematite cubes in a rotating magnetic field-experiments, J. Magn. Magn. Mater. 500, 166404 (2020).

[25] E. S. Bililign, F. B. Usabiaga, Y. A. Ganan, V. Soni, S. Magkiriadou, M. J. Shelley, D. Bartolo, and W. Irvine, Chiral crystals self-knead into whorls, arXiv:2102.03263.

[26] P. Tierno, R. Muruganathan, and T. M. Fischer, Viscoelasticity of Dynamically Self-Assembled Paramagnetic Colloidal Clusters, Phys. Rev. Lett. 98, 028301 (2007).

[27] N. Osterman, I. Poberaj, J. Dobnikar, D. Frenkel, P. Ziherl, and D. Babić, Field-Induced Self-Assembly of Suspended Colloidal Membranes, Phys. Rev. Lett. 103, 228301 (2009).

[28] J. Yan, S. C. Bae, and S. Granick, Rotating crystals of magnetic janus colloids, Soft Matter 11, 147 (2015).

[29] D. Du, M. Doxastakis, E. Hilou, and S. L. Biswal, Twodimensional melting of colloids with long-range attractive interactions, Soft Matter 13, 1548 (2017).

[30] A. T. Pham, Y. Zhuang, P. Detwiler, J. E. S. Socolar, P. Charbonneau, and B. B. Yellen, Phase diagram and aggregation dynamics of a monolayer of paramagnetic colloids, Phys. Rev. E 95, 052607 (2017).

[31] G. Helgesen, P. Pieranski, and A. T. Skjeltorp, Nonlinear Phenomena in Systems of Magnetic Holes, Phys. Rev. Lett. 64, 1425 (1990).

[32] J. Černák and G. Helgesen, Aggregation of magnetic holes in a rotating magnetic field, Phys. Rev. E 78, 061401 (2008).

[33] See Supplemental Material at http://link.aps.org/supplemental/ 10.1103/PhysRevResearch.3.L042021 for four videos as support of the experimental results and one .pdf file with more details of the experimental system and numerical simulations.

[34] S. Jäger, H. Stark, and S. H. L. Klapp, Dynamics of cluster formation in driven magnetic colloids dispersed on a monolayer, J. Phys.: Condens. Matter 25, 195104 (2013).

[35] F. Martinez-Pedrero, A. Cebers, and P. Tierno, Orientational dynamics of colloidal ribbons self assembled from microscopic magnetic ellipsoids, Soft Matter 12, 3688 (2016).

[36] P. Lenz, J.-F. Joanny, F. Jülicher, and J. Prost, Membranes with Rotating Motors, Phys. Rev. Lett. 91, 108104 (2003).

[37] Y. Fily, A. Baskaran, and M. C. Marchetti, Cooperative selfpropulsion of active and passive rotors, Soft Matter 8, 3002 (2012). 
[38] F. Martinez-Pedrero, A. Ortiz-Ambriz, I. Pagonabarraga, and P. Tierno, Colloidal Microworms Propelling Via a Cooperative Hydrodynamic Conveyor Belt, Phys. Rev. Lett. 115, 138301 (2015).

[39] A. J. Bray, Theory of phase-ordering kinetics, Adv. Phys. 51, 481 (2002).

[40] S. Puri and V. Wadhawan, Kinetics of Phase Transitions (CRC Press, Boca Raton, 2009).

[41] I. Theurkauff, C. Cottin-Bizonne, J. Palacci, C. Ybert, and L. Bocquet, Dynamic Clustering in Active Colloidal Suspensions with Chemical Signaling, Phys. Rev. Lett. 108, 268303 (2012).

[42] I. Buttinoni, J. Bialké, F. Kümmel, H. Löwen, C. Bechinger, and T. Speck, Dynamical Clustering and Phase Separation in Suspensions of Self-Propelled Colloidal Particles, Phys. Rev. Lett. 110, 238301 (2013).

[43] J. Palacci, S. Sacanna, A. P. Steinberg, D. J. Pine, and P. M. Chaikin, Living crystals of light-activated colloidal surfers, Science 339, 936 (2013).

[44] D. Levis and L. Berthier, Clustering and heterogeneous dynamics in a kinetic monte carlo model of self-propelled hard disks, Phys. Rev. E 89, 062301 (2014).

[45] J. Bialké, T. Speck, and H. Löwen, Active colloidal suspensions: Clustering and phase behavior, J. Non-Cryst. Solids 407, 367 (2015)

[46] F. Ginot, I. Theurkauff, F. Detcheverry, C. Ybert, and C. CottinBizonne, Aggregation-fragmentation and individual dynamics of active clusters, Nat. Commun. 9, 696 (2018).

[47] B. Liebchen and D. Levis, Collective Behavior of Chiral Active Matter: Pattern Formation and Enhanced Flocking, Phys. Rev. Lett. 119, 058002 (2017).

[48] D. Levis and B. Liebchen, Micro-flock patterns and macroclusters in chiral active brownian disks, J. Phys.: Condens. Matter 30, 084001 (2018).

[49] H. Massana-Cid, F. Meng, D. Matsunaga, R. Golestanian, and P. Tierno, Tunable self-healing of magnetically propelling colloidal carpets, Nat. Commun. 10, 2444 (2019).

[50] Q. Yang, H. Zhu, P. Liu, R. Liu, Q. Shi, K. Chen, N. Zheng, F. Ye, and M. Yang, Topologically Protected Transport of Cargo in a Chiral Active Fluid Aided by Odd-Viscosity-Enhanced Depletion Interactions, Phys. Rev. Lett. 126, 198001 (2021).

[51] A. Souslov, A. Gromov, and V. Vitelli, Anisotropic odd viscosity via a time-modulated drive, Phys. Rev. E 101, 052606 (2020).
[52] J. R. Blake and A. T. Chwang, Fundamental singularities of viscous flow, J. Eng. Math. 8, 23 (1974).

[53] J. Happel and H. Brenner, Low Reynolds number Hydrodynamics (Noordhoff, Leyden, 1965).

[54] B. A. Grzybowski, H. A. Stone, and G. M. Whitesides, Dynamic self-assembly of magne-tized, millimetre-sized objects rotating at a liquidair interface, Nature (London) 405, 1033 (2000).

[55] E. Climent, K. Yeo, M. R. Maxey, and G. E. Karniadakis, Dynamic self-assembly of spinningparticles, J. Fluids Eng. 129, 379 (2007).

[56] R. Di Leonardo, A. Búzás, L. Kelemen, G. Vizsnyiczai, L. Oroszi, and P. Ormos, Hydrodynamic Synchronization of Light Driven Microrotors, Phys. Rev. Lett. 109, 034104 (2012).

[57] G. Kokot, D. Piet, G. Whitesides, I. S. Aranson, and A. Snezhko, Emergence of reconfigurable wires and spinners via dynamic self-assembly, Sci. Rep. 5, 9528 (2015).

[58] G. Kokot, S. Das, R. Winkler, G. Gompper, I. Aranson, and A. Snezhko, Active turbulence in a gas of self-assembled spinners, Proc. Nat. Acad. Sci. USA 114, 12870 (2017).

[59] I. Riedel, K. Kruse, and J. Howard, A self-organized vortex array of hydrodynamically entrained sperm cells, Science $\mathbf{3 0 9}$, 300 (2005)

[60] K. Drescher, K. C. Leptos, I. Tuval, T. Ishikawa, T. J. Pedley, and R. E. Goldstein, Dancing Volvox: Hydrodynamic Bound States of Swimming Algae, Phys. Rev. Lett. 102, 168101 (2009).

[61] D. R. Brumley, M. Polin, T. J. Pedley, and R. E. Goldstein, Hydrodynamic Synchronization and Metachronal Waves on the Surface of the Colonial Alga Volvox Carteri, Phys. Rev. Lett. 109, 268102 (2012).

[62] A. P. Petroff, X.-L. Wu, and A. Libchaber, Fast-Moving Bacteria Self-Organize Into Active Two-Dimensional Crystals of Rotating Cells, Phys. Rev. Lett. 114, 158102 (2015).

[63] C. J. Pierce, H. Wijesinghe, E. Mumper, B. H. Lower, S. K. Lower, and R. Sooryakumar, Hydrodynamic Interactions, Hidden Order, and Emergent Collective Behavior in an Active Bacterial Suspension, Phys. Rev. Lett. 121, 188001 (2018).

[64] N. Oppenheimer, D. B. Stein, and M. J. Shelley, Rotating Membrane Inclusions Crystallize Through Hydrodynamic and Steric Interactions, Phys. Rev. Lett. 123, 148101 (2019). 